\title{
OCULOCEREBRORENAL SYNDROME OF LOWE
}

\section{Magnetic resonance imaging findings in the first six years of life}

\author{
Arnolfo de Carvalho-Neto', Sergio Eiji Ono', Georgina de Melo Cardoso', \\ Mara Lucia Schmitz Ferreira Santos², Izabela Celidonio ${ }^{3}$
}

The oculocerebrorenal syndrome of Lowe (OCRL), was first recognized as a distinct disease in 1952 by Drs. Lowe, Terrey and MacLachlan at Massachusetts General Hospital, in Boston, USA, describing three male children with organic aciduria, decreased renal ammonia production, hydrophtalmos and mental retardation'. The X-linked recessive inheritance pattern was recognized first by LeFebvre ${ }^{2}$. It is present in all races, with a predominance in those of Caucasian and Asian ancestries. Rarely females are affect$\mathrm{ed}^{3}$. It is a very rare disease, with estimated prevalence in the general population of 1 in 500,000. In USA the Lowe Syndrome Association (LSA) documented 190 living patients in the year $2000(0.67 \times \text { million inhabitants })^{4}$.

It is caused by a mutation in the gene encoding oculocerebrorenal-Lowe protein (OCRL1), isolated in 1992, linked to the Xq24-q26 region of the X chromosome ${ }^{2,4-6}$. Approximately $60 \%$ of OCRL patients demonstrate a loss of OCRL1 gene expression ${ }^{2}$, and the definitive laboratory test, that can be used for prenatal diagnosis, is the biochemical assay for deficiency of phosphatidylinositol 4,5biphosphate 5-phosphate in cultured fibroblasts ${ }^{3}$.

The classic triad of eye, central nervous system, and kidney involvement are required for the diagnosis of Lowe's syndrome $e^{4}$. Cataract is present at birth in all patients and glaucoma is detected within the first year of life. Hypotonia compromises suction and causes serious respiratory problems in the first period of life. Motor development is retarded and mental retardation is moderate or severe in almost all cases. Obsessivecompulsive behavior is typical. Seizure is seen in approximately $50 \%$ of the patients over 18 years old. Renal disease is primarily characterized by renal Fanconi syndrome but many children are asymptomatic at birth. Renal involvement is initially related to bicarbonate, salt and water wasting, causing failure to thrive. Later, a significant number of patients devel- op chronic renal failure. The treatment includes cataract extraction, glaucoma control, physical and speech therapy, drugs addressed to behavioral problems and correction of the renal (tubular acidosis) and consequent bone diseases (rickets). Life span rarely exceeds 40 years ${ }^{4}$.

Brain magnetic resonance imaging (MRI) may show two patterns of lesions: hyperintensities on T2-weighted images, and periventricular cystic lesions ${ }^{2,4-9}$. The present case reports these findings in a time interval of 4 years, showing that initially the hyperintensities are seen, and afterwards, the cystic images. This pattern would help the radiologist and pediatric neurologist to reinforce the clinical diagnosis, as these patterns of images can be seen in other conditions.

\section{CASE}

The present case report was approved by the Ethics Committee of the Medical Center and has an informed consent given by the parents of the child. The patient had congenital bilateral cataracts and underwent surgery at the age of 4 months. Afterwards he developed psychomotor retardation, hypotonia, hypoglicemia, dysmorphic facial features, difficulty in swallowing and severe behavior disturbances (irritability and self-mutilation), which progressively worsened. Although he never had seizures, his electroencephalogram exam showed frontal lobe discharges. Renal impairment was detected at the age of 4 when laboratory tests revealed urinary calcium wasting. On the basis of the clinical course and the laboratory parameters, Lowe syndrome was diagnosed.

\section{DISCUSSION}

The patient had a clinical diagnosis of OCRL syndrome with most of the known related symptoms. The first cranial MR examination (Fig 1), done at 21 months of age, consisted of white matter abnormalities. There were bilat-

\section{SÍNDROME OCULOCEREBRORRENAL DE LOWE: ACHADOS EVOLUTIVOS DE IMAGEM DE RESSONÂNCIA MAGNÉTICA NOS PRIMEIROS SEIS ANOS DE VIDA}

Imaging Center: DAPI - Diagnóstico Avançado por Imagem, Curitiba PR, Brazil: 'Radiologist, Medical Center: Hospital Pequeno Príncipe, Curitiba PR, Brazil: ${ }^{2}$ Child Neurologist; ${ }^{3}$ Child Neurologist Resident.

Received 5 August 2008, received in final form 9 December 2008. Accepted 2 March 2009.

Dr. Sergio Eiji Ono - Rua Brigadeiro Franco 122 - 80430-120 Curitiba PR - Brasil. E-mail: ono.sergio@gmail.com 


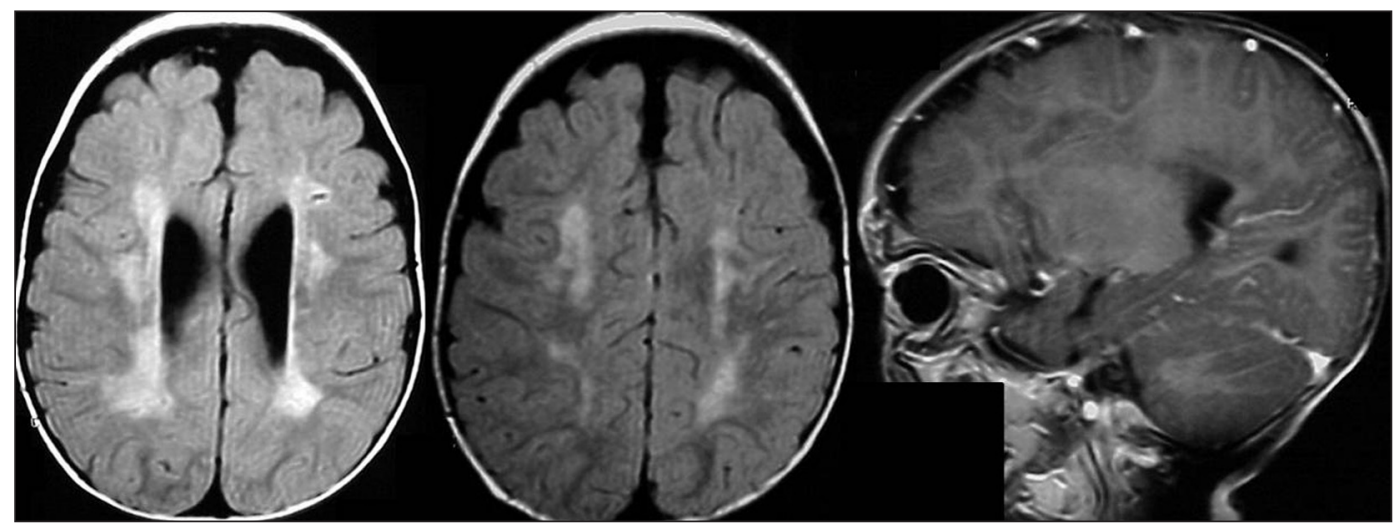

Fig 1. MRI examination at 21 months of age. Axial FLAIR (fluid-attenuated inversion recovery) and sagital T7-weighted post gadolinium (MR contrast agent). T2 weighted images show white matter hyperintensities in the periventricular and centrum semiovale regions.

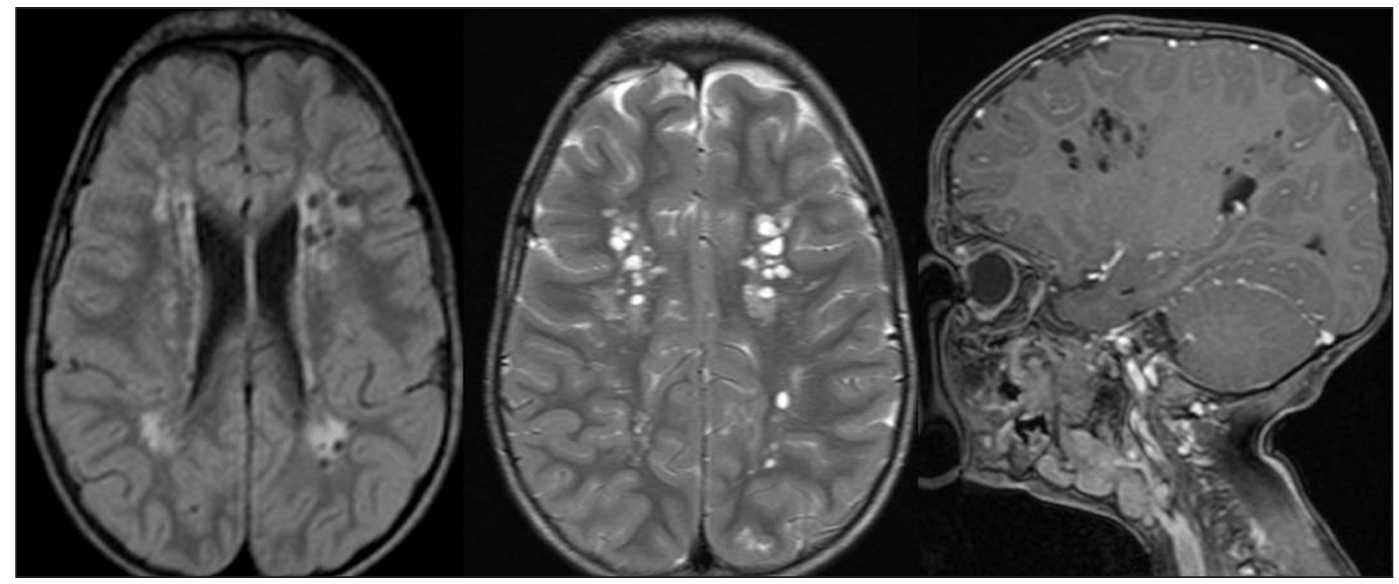

Fig 2. MRI at six years of age. Axial FLAIR and T2-weighted fast spin-echo, and sagital T7-weighted post gadolinium images showing the appearance of cysts (perivascular lacunes).

eral hyperintensities seen on T2-weighted images in the periventricular white matter and in the centrum semiovale. These are nonspecific findings, but in the context of OCRL syndrome, this is considered gliosis ${ }^{8}$. The second examination (Fig 2), done four years later, at six years of age, showed the appearance of cysts in the periventricular white matter that corresponds to perivascular lacunes in OCRL.

Reports in the past attributed the hyperintensities to demyelination ${ }^{6,7}$ or gliosis ${ }^{8}$, and not necessarily correlated with the severity of clinical manifestations ${ }^{7}$. Recent studies and neurophatological data show evidences that these are due to gliosis. Schneider and Sener ${ }^{2,8}$ described cases with proton MR spectroscopy reporting prominent myoinositol peaks, a glial marker, suggesting the presence of gliosis. But at the same time, it is not clear if these peaks could represent accumulation of phosphatidylinositol 4,5-biphosphate, a metabolite which is not degraded in patients with Lowe syndrome ${ }^{2}$. The gene product domain affected in OCRL patients is an inositol polyphos- phate 5-phosphatase, located in the Golgi apparatus, that catalyzes the hydrolysis of the 5-position phosphate and controls cellular levels of phosphatidylinositol 4,5-biphosphate (Ptdlns 4,5-P2), a metabolite involved in Golgi vesicular transport. It has been postulated that the enzymatic deficiency lead the accumulation of Ptdlns 4,5-P2 in lysosomal membranes and therefore increased extracellular release of lysosomal enzymes. This would cause developmental defects in the lens and abnormal renal and neurological functions. In CNS the accumulation of lysosomal products can lead to dilatation of perivascular spaces, whereas extracellular release of lysosomal enzymes can lead to toxic gliotic reactions ${ }^{2}$.

Neuropathological findings are limited, describing brain weight usually below normal, atrophy, ventricular enlargement and thinning of the corpus calosum. The meninges are diffusely fibrotic without inflammatory changes. The most frequent finding in the white matter is gliosis, mainly in the centrum semiovale and in the periventricular region. Spongy changes have been observed, frequently 
associated with perivascular lacunes. Active myelin breakdown (demyelination) has never been reported ${ }^{3}$.

MR findings alone are sometimes nonspecific, and without clinical data the radiologist's report could be misleading. Diffuse white matter abnormalities can be attributed to several diseases and conditions. The OCRL syndrome is diagnosed based on clinical and laboratory data, but this article may help the neuroradiologist to reinforce the diagnosis. The two MR findings seen in OCRL patients - hyperintensities and cysts - are well described in the literature, but to the best of our knowledge, there are virtually no reports describing the sequential appearance of these findings on MRI.

\section{REFERENCES}

1. Lowe CU, Terrey M, MacLachan EA. Organic-aciduria, decreased renal ammonia production, hydrophthalmos, and mental retardation; a clinical entity. Am J Dis Child 1952;83:164-184.

2. Schneider JF, Boltshauser E, Neuhaus TJ, Rauscher C, Martin
E. MRI and proton spectroscopy in Lowe syndrome. Neuropediatrics 2001;32:45-48.

3. Knaap MS, Valk J. Lowe syndrome. Magnetic resonance of myelination and myelin disorders. Third edition. New York: Springer 2005:387-391.

4. Loi M. Lowe syndrome. Orphanet J Rare Dis 2006;1:16.

5. Pueschel SM, Brem AS, Nittoli P. Central nervous system and renal investigations in patients with Lowe syndrome. Child's Nerv System 1992;8:45-48.

6. Carroll WJ, Woodruff WW, Cadman TE. MR findings in oculocerebrorenal syndrome. AJNR 1993;14:449-451.

7. Ono J, Harada K, Mano T, Yamamoto T, Okada S. MR findings and neurological manifestations in Lowe oculocerebrorenal syndrome. Pediatr Neurol 1996;4:162-164.

8. Sener RN. Lowe syndrome: proton MR spectroscopy, and diffusion MR imaging. J Neuroradiol 2004; 31:238-240.

9. Demmer LA, Wippold II FJ, Dowton SB. Periventricular white matter cystic lesions in Lowe (Oculocerebrorenal) syndrome. Pediatric Radiology 1992;22:76-77. 